\title{
The Association between Metabolic Syndrome-Related Indicators and Ten-Year Risk of Cardiovascular Disease: An Epidemiologic Assessment
}

\author{
Tsan Yang, ${ }^{1}$ Chien-An Sun, ${ }^{2}$ Ching-Chuan Chen, ${ }^{3}$ Szu-Hsien Wu, ${ }^{4}$ \\ Tieh-Chi Chung, ${ }^{5}$ and Yu-Ching $\mathrm{Chou}^{6}$
}

${ }^{1}$ Department of Health Business Administration, Meiho University, Pingtung 91202, Taiwan

${ }^{2}$ Department of Public Health, College of Medicine, Fu Jen Catholic University, New Taipei City 24205, Taiwan

${ }^{3}$ Department of Food and Nutrition, Meiho University, Pingtung 91202, Taiwan

${ }^{4}$ Respiratory Care Center, Kaohsiung Armed Forces General Hospital, Kaohsiung City 80284, Taiwan

${ }^{5}$ Graduate Institute of Health Care, Meiho University, Pingtung 91202, Taiwan

${ }^{6}$ School of Public Health, National Defense Medical Center, Taipei City 11490, Taiwan

Correspondence should be addressed to Tsan Yang, tsan.yang@msa.hinet.net

Received 1 September 2011; Accepted 18 October 2011

Academic Editor: K. Søgaard

Copyright (๑) 2012 Tsan Yang et al. This is an open access article distributed under the Creative Commons Attribution License, which permits unrestricted use, distribution, and reproduction in any medium, provided the original work is properly cited.

Objectives. This study explored the association between metabolic syndrome-related indicators and the ten-year risk for cardiovascular disease (CVD) in the middle-aged and elderly population. Methods. Data were collected from residents in Southern Taiwan through a health screening program, carried out from March 2007 to May 2008. The present study used the risk assessment tool of the Framingham Heart Study and adopted $\leqq 10 \%$ as low risk, $11 \sim 20 \%$ as medium risk, and $>20 \%$ as high risk for CVD. Results. We found that subjects with abdominal obesity and hypertension had significantly higher 10-year risk for CVD than those who had normal waist circumference and blood pressure ( $O R=2.07$ and 24.52, resp.). Subjects with hypertriglyceridemia were 1.86 times more at risk for developing medium risk of CVD than those who had normal triglyceride levels. Furthermore, individuals with reduced high-density lipoprotein cholesterol (HDL-C) had significantly higher 10-year risk for CVD than those who had normal HDL-C. Conclusions. Metabolic syndrome-related indicators were positively associated with 10-year risk for CVD. Waist circumference (WC), blood pressure, triglyceride and HDL-C might be good indicators for predicting the risk of CVD for middle-aged and elderly populations.

\section{Introduction}

Metabolic syndrome is the concurrence in an individual of interrelated risk factors for CVD and diabetes. These factors include hyperglycemia, raised blood pressure, elevated triglyceride concentrations, reduced HDL-C, and obesity (particularly central adiposity) [1]. Studies have demonstrated that the syndrome is common when these risk factors are present and that its global prevalence is increasing. The trend relates largely to the increase in obesity and sedentary lifestyles [2]. Consequently, metabolic syndrome represents a global public health problem.
Metabolic syndrome has been recognized as a major risk factor for the development of CVD and type 2 diabetes worldwide [3]. Previous research indicated that the risk for coronary heart disease (CHD) and stroke was increased threefold in subjects with metabolic syndrome $(P<.001)$. Cardiovascular mortality was also markedly increased in subjects with metabolic syndrome (12.0 versus $2.2 \%, P<$ .001) [4]. Dekker et al. studied the European population and concluded that the metabolic syndrome was associated with an approximately twofold increased risk of incident cardiovascular morbidity and mortality [5]. Another study showed that patients with even one or two metabolic 
syndrome components were at increased risk for mortality from CHD and CVD. Moreover, metabolic syndrome overall more strongly predicts $\mathrm{CHD}, \mathrm{CVD}$, and total mortality than its individual components [6]. Similarly, the risk of incident CVD increased in conjunction with rising numbers of the components of metabolic syndrome; $2.5 \%$ of individulals with one component developed CVD, whereas $14.9 \%$ of those who had four or more components developed the disease [7].

However, the utility of the metabolic syndrome in predicting risk for the CVD requires further understanding. Previous studies have documented that under fixed body mass index (BMI), metabolic risk (e.g., insulin resistance, diabetes, and cardiovascular disease) was significantly higher in Asians than in Caucasians [8]. To our knowledge, few Taiwanese studies have been conducted with middle-aged and elderly populations living in the community. Fewer still have utilized the Framingham Heart Study as a risk assessment tool to evaluate metabolic syndrome as a whole, as well as its individual components as indicators of CVD risk. The results of such studies could be used to inform screening programs and as a reference for management of metabolic syndrome and its individual components in the middle-aged and elderly populations. Accordingly, we assessed the association between components of metabolic syndrome and 10-year risk of CVD in the middle-aged and elderly population in Southern Taiwan.

\section{Methods}

The study design was cross-sectional, and research protocol was approved by the Institutional Review Board at the Meiho University, Pingtung, Taiwan. Participants were residents of Pingtung County in Southern Taiwan and were over 40 years of age. Data were collected through a health screening program, carried out from March 2007 to May 2008. A total of 859 adults participated in the survey, but 28 individuals were excluded because of missing information on the evaluation items for the 10-year risk of CVD. The final sample, therefore, consisted of 831 adults.

\section{Data Collection}

A self-administered questionnaire was used to collect information on sociodemographic characteristics of the study participants and their medical history and lifestyle behaviors. Physical examinations were conducted in the health center of a local teaching hospital. In addition, each participant's blood pressure was recorded, as were anthropometric measurements including height, weight, and waist circumference (WC). Height was measured to the nearest $0.1 \mathrm{~cm}$ without shoes using a stadiometer; weight was measured in light clothing without shoes using a beam balance scale and was recorded to the nearest $0.1 \mathrm{~kg}$; waist circumference was measured to the nearest $0.5 \mathrm{~cm}$ above the iliac crests and below the lowest rib margin at minimal respiration in a standing position. Blood pressures were taken twice with the right arm, with the subject in a seated position, using an electronic automatic blood pressure device (model 3M
Littmann Classic $2 \mathrm{~S}$. Stethoscope). If the two readings varied by more than $10 \mathrm{mmHg}$, an additional measurement was taken, and the average of the two closest readings of blood pressures was used in the analysis. Fasting venous blood samples were collected from each subject for a battery of biochemistry analyses; the study subjects had fasted for 10 hours prior to the blood collection. The blood samples were placed in a $4^{\circ} \mathrm{C}$ ice bucket, delivered to the laboratory within four hours, kept in the refrigerator, and the analysis was completed within a week. The laboratory analyses included fasting plasma glucose (FPG), triglycerides (TG), and HDLC.

Metabolic syndrome status was defined according to the criteria set in the Third Report of the National Cholesterol Education Program (NCEP) Expert Panel on Detection, Evaluation, and Treatment of High Blood Cholesterol in Adults (Adult Treatment Panel III, ATP-III) [9].

We utilized previously established modifications for Asian populations, using waist circumference cut-points [10]. Any three of the following five criteria were grounds for identifying metabolic syndrome:

(1) abdominal obesity: waist circumference (WC) $\geqq 90$ $\mathrm{cm}$ in men and $\geqq 80 \mathrm{~cm}$ in women;

(2) raised TG: $\geqq 150 \mathrm{mg} / \mathrm{dL}$;

(3) reduced HDL-C: HDL-C $<40 \mathrm{mg} / \mathrm{dL}$ in men and $<50 \mathrm{mg} / \mathrm{dL}$ in women;

(4) hypertension: blood pressure of at least $130 / 85 \mathrm{~mm}$ $\mathrm{Hg}$ or taking antihypertensive medication; and

(5) raised $\mathrm{FPG} \geqq 110 \mathrm{mg} / \mathrm{dL}$ and/or taking anti-glycemic medication.

\section{Statistical Analysis}

Risk assessment for determining the 10-year risk for developing CVD was performed using Framingham risk scoring. The risk factors included in the Framingham calculation were sex, age, total cholesterol, HDL-C, smoking habits, systolic blood pressure, and medication taken to treat high blood pressure [9]. Our study adopted $\leqq 10 \%$ as low risk, $11 \sim 20 \%$ as medium risk, and $>20 \%$ as high risk for CVD. Associations between metabolic syndrome-related risk factors and their predicted 10-year risk for developing CVD were evaluated by $\chi^{2}$ tests and by odds ratios (ORs) and their $95 \%$ confidence intervals (CIs) derived from logistic regression analyses. All statistical tests were two sided, and values of $P<$ 0.05 were considered statistically significant. The statistical analyses were performed with Statistical Package for the Social Sciences v.17.0 (SPSS Chicago, IL).

\section{Results}

Of the 831 research subjects, 341 were men and 490 were women; the mean age of the sample ( \pm standard deviation) was $65.39 \pm 10.93$ years. A total of 269 subjects were found to have metabolic syndrome, a prevalence rate of $32.4 \%$. We found that subjects with metabolic syndrome were 
TABle 1: Prevalence rates for metabolic syndrome-related indicators among study participants.

\begin{tabular}{|c|c|c|}
\hline Variable & Number & Percentage \\
\hline \multicolumn{3}{|l|}{ Waist circumference $(\mathrm{cm})$} \\
\hline Normal & 504 & 60.6 \\
\hline Abdominal obesity ${ }^{\mathrm{a}}$ & 327 & 39.4 \\
\hline \multicolumn{3}{|l|}{ Blood pressure $(\mathrm{mmHg})$} \\
\hline Normal & 268 & 32.3 \\
\hline Hypertension $^{\mathrm{b}}$ & 563 & 67.7 \\
\hline \multicolumn{3}{|c|}{ Fasting plasma glucose $(\mathrm{mg} / \mathrm{dL})$} \\
\hline Normal & 636 & 76.5 \\
\hline Hyperglycemia $^{c}$ & 195 & 23.5 \\
\hline \multicolumn{3}{|l|}{ Triglycerides (mg/dL) } \\
\hline Normal & 606 & 72.9 \\
\hline Hypertriglyceridemia $^{\mathrm{d}}$ & 225 & 27.1 \\
\hline \multicolumn{3}{|l|}{ HDL-C (mg/dL) } \\
\hline Normal & 506 & 60.9 \\
\hline Low HDL-C ${ }^{e}$ & 325 & 39.1 \\
\hline \multicolumn{3}{|l|}{ Metabolic syndrome } \\
\hline Not present & 562 & 67.6 \\
\hline Present ${ }^{\mathrm{f}}$ & 269 & 32.4 \\
\hline \multicolumn{3}{|c|}{$\begin{array}{l}\text { a Male: } \geq 90 \mathrm{~cm} \text {; female: } \geqq 80 \mathrm{~cm} \text {. } \\
\mathrm{b} \geq 130 / 85 \mathrm{mmHg} \text { or taking antihypertensive medication. } \\
\mathrm{c} \geq 110 \mathrm{mg} / \mathrm{dL} \text { or taking medication for lowering blood glucose. } \\
\mathrm{d} \geq 150 \mathrm{mg} / \mathrm{dL} \text {. } \\
\text { e Male: }<40 \mathrm{mg} / \mathrm{dL} \text {; female }<50 \mathrm{mg} / \mathrm{dL} \text {. } \\
\text { f Definition of metabolic syndrome as per NCEP ATP III, modified for Asiar } \\
\text { populations. }\end{array}$} \\
\hline
\end{tabular}

significantly overrepresented in the medium- and high-risk groups for 10 -year risk of CVD $(P<.003)$.

The prevalence of the metabolic syndrome-related indicators was as follows: abdominal obesity 39.4\%; hypertension $67.7 \%$; hyperglycemia $23.5 \%$; hypertriglyceridemia 27.1\%; reduced HDL-C 39.1\% (Table 1). As expected, significant associations were found between demographic characteristics, lifestyle behaviors, metabolic syndrome-related indicators, and predicted 10-year risk for CVD (Table 2). For example, compared to participants with normal blood pressure, those who had a diagnosis of hypertension were more likely to be in the higher predicted 10-year risk groupings ( $11-20 \%$ and $\geqq 20 \%)$. Similar results were found for metabolic syndrome overall as well as its individual components, as listed in Table 2.

Multiple logistic regression was used to assess the association between metabolic syndrome-related indicators and 10year risk for CVD (Table 3). Adjustments were made for sex, age, smoking, alcohol consumption, and betel-nut chewing. The results revealed that among the medium-risk and highrisk groups, four predicted variables showed statistically significant relationships with 10 -year risk for CVD. These four variables were WC, blood pressure, TG, and HDL-C.

The remaining syndrome-related indicator variables did not show significant associations with CVD risk. Specifically, when we used the low-risk group as the reference group and adjusted for potential confounding factors, subjects with abdominal obesity and elevated blood pressure had a higher 10-year risk for CVD than those who had normal WC and blood pressure. The adjusted odds ratios were 2.07 (95\% CI $=1.04-4.13)$ and 24.52 (95\% CI $=9.10-66.09)$, respectively. For subjects with elevated blood pressure, the odds ratio for the medium-risk group was $3.12(95 \% \mathrm{CI}=$ 1.75-5.55) compared to the low-risk group ( $\leqq 10 \%)$. The risk of developing a medium-risk CVD was 1.86 times $(95 \% \mathrm{CI}$ = 1.11-3.13) higher for subjects with hypertriglyceridemia, compared to subjects with normal TG. Similar results were observed among the subjects with low HDL-C.

\section{Discussion}

The purpose of this study was to explore the association between metabolic syndrome-related indicators and the predicted 10-year risk for CVD in the middle-aged and elderly population in Southern Taiwan. We observed that metabolic syndrome-related indicators were significantly associated with an elevated risk of CVD in these population groups. Specifically, individuals with abdominal obesity, hypertension, hypertriglyceridemia, and reduced HDL-C levels were more likely to develop CVD in 10 years.

Regarding the distributions of individual components of metabolic syndrome, the present study revealed that the prevalence rates of abdominal obesity, hypertension, hyperglycemia, hypertriglyceridemia, and reduced HDL-C were $39.4 \%, 67.7 \%, 23.5 \%, 27.1 \%$, and $39.1 \%$, respectively. A previous population-based study in Taiwan showed that $29.7 \%$ of subjects had hypertension; the same study found that the prevalence rates of hyperglycemia, hypertriglyceridemia, and reduced $\mathrm{HDL}-\mathrm{C}$ are $18.5 \%, 27.5 \%$, and $23.4 \%$, respectively [11].

The prevalence of metabolic disorders was higher in Western populations, with prevalence rates for hypertension, hyperglycemia, hypertriglyceridemia, and reduced HDL-C being reported as $79 \%, 38 \%, 44 \%$, and $42 \%$, respectively [12].The variations between studies for prevalence rates of metabolic syndrome-related indicators may be because of differences in age and racial distributions or lifestyle behaviors.

The prevalence of metabolic syndrome in our study was $32.4 \%$. This figure is higher than that of a previous study in Taiwan using the same criteria, namely, the NCEP-ATP III criteria with the Asian modification of waist circumference; in that study, the prevalence was found to be $24.2 \%$ [13]. Again, using the same criteria and a Taiwanese sample, Lai et al. conducted a cross-sectional study and found the prevalence of metabolic syndrome to be $44.1 \%$ [14]. The discrepancy in these three prevalence rates for metabolic syndrome may be because of age characteristics of the various participants.

Few previous studies had focused directly on 10-year risk of developing CVD among individuals with metabolic syndrome. In our study, we found subjects who were male, older (age 65 and over), smokers, alcohol consumers, and betel-nut chewers to be over-represented in the medium and high-risk groups for the 10-year CVD risk. Furthermore, after adjusting the analysis for sex, age, smoking, alcohol 
TABLE 2: Demographic characteristics of study participants, metabolic syndrome-related indicators, and predicted 10-year risk for CVD $(n=831)$.

\begin{tabular}{|c|c|c|c|c|}
\hline \multirow{2}{*}{ Variable } & \multicolumn{4}{|c|}{ 10-year risk for CVD } \\
\hline & $\leqq 10 \%$ & $11-20 \%$ & $>20 \%$ & $P^{*}$ value \\
\hline Sex & & & & $<.001$ \\
\hline Male & $72(21.1)$ & $161(47.2)$ & $108(31.7)$ & \\
\hline Female & $424(86.5)$ & $56(11.4)$ & $10(2.0)$ & \\
\hline Age & & & & $<.001$ \\
\hline$<65$ & $285(82.1)$ & $49(14.1)$ & $13(3.7)$ & \\
\hline$\geqq 65$ & $211(43.6)$ & $168(34.7)$ & $105(21.7)$ & \\
\hline Smoking & & & & $<.001$ \\
\hline $\mathrm{No}^{\mathrm{a}}$ & $469(64.7)$ & $167(23.0)$ & $89(12.3)$ & \\
\hline Yes & $27(25.5)$ & $50(47.2)$ & $29(27.4)$ & \\
\hline Alcohol consumption & & & & $<.001$ \\
\hline $\mathrm{No}^{\mathrm{b}}$ & $454(63.1)$ & $170(23.6)$ & $96(13.3)$ & \\
\hline Yes & $42(37.8)$ & $47(42.3)$ & $22(19.8)$ & \\
\hline Betel-nut chewing & & & & $<.001$ \\
\hline $\mathrm{No}^{\mathrm{c}}$ & $482(61.3)$ & $194(24.7)$ & $110(14.0)$ & \\
\hline Yes & $14(31.1)$ & $23(51.1)$ & $8(17.8)$ & \\
\hline Regular exercise & & & & .183 \\
\hline No & $245(59.0)$ & $118(28.4)$ & $52(12.5)$ & \\
\hline $\mathrm{Yes}^{\mathrm{d}}$ & $251(60.3)$ & $99(23.8)$ & $66(15.9)$ & \\
\hline Waist circumference $(\mathrm{cm})$ & & & & .652 \\
\hline Normal & $295(58.5)$ & $137(27.2)$ & $72(14.3)$ & \\
\hline Abdominal obesity & $201(61.5)$ & $80(24.5)$ & $46(14.1)$ & \\
\hline Blood pressure (mmHg) & & & & $<.001$ \\
\hline Normal & $204(76.1)$ & $57(21.3)$ & $7(2.6)$ & \\
\hline Hypertension $^{\mathrm{f}}$ & $292(51.9)$ & $160(28.4)$ & $111(19.7)$ & \\
\hline Fasting plasma glucose $(\mathrm{mg} / \mathrm{dL})$ & & & & .023 \\
\hline Normal & $396(62.3)$ & $156(24.5)$ & $84(13.2)$ & \\
\hline Hyperglycemiag $^{g}$ & $100(51.3)$ & $61(31.3)$ & $34(17.4)$ & \\
\hline HDL-C(mg/dL) & & & & .026 \\
\hline Normal & $298(58.9)$ & $146(28.9)$ & $62(12.3)$ & \\
\hline Low high-density lipoprotein cholesterol $^{\text {h }}$ & $198(60.9)$ & $71(21.8)$ & $56(17.2)$ & \\
\hline Triglycerides (mg/dL) & & & & .009 \\
\hline Normal & $381(62.9)$ & $145(23.9)$ & $80(13.2)$ & \\
\hline Hypertriglyceridemia ${ }^{\mathrm{i}}$ & $115(51.1)$ & $72(32.0)$ & $38(16.9)$ & \\
\hline Metabolic syndrome & & & & .003 \\
\hline Not present & $354(63.0)$ & $143(25.4)$ & $65(11.6)$ & \\
\hline Present $^{j}$ & $142(52.8)$ & $74(27.5)$ & $53(19.7)$ & \\
\hline
\end{tabular}

${ }^{a}$ Never smoked or has quit.

${ }^{\mathrm{b}}$ Never drinks or has quit.

${ }^{\mathrm{c}}$ Never chews betel nut, or has quit.

d $\geq$ at least 3 times/week for at least 30 mins/session.

'Male: $\geq 90 \mathrm{~cm}$; female: $\geq 80 \mathrm{~cm}$.

$\mathrm{f} \geqq 130 / 85 \mathrm{mmHg}$ or taking antihypertensive medication.

$\mathrm{g} \geqq 110 \mathrm{mg} / \mathrm{dL}$ or taking medication to lower blood glucose.

${ }^{\mathrm{h}}$ Male: $<40 \mathrm{mg} / \mathrm{dL}$; female $<50 \mathrm{mg} / \mathrm{dL}$.

$\mathrm{i} \geqq 150 \mathrm{mg} / \mathrm{dL}$.

jMetabolic syndrome definition of NCEP ATP III with modification for Asian populations. ${ }^{*}$ The Chi-square (two-tailed) test was used to compare the variables, with a significance level of $\alpha=.05$. 
TABLE 3: Multiple logistic regression analysis of the association between metabolic syndrome-related indicators and predicted 10-year risk for CVD.

\begin{tabular}{lcr}
\hline & \multicolumn{2}{c}{ 10-year risk for CVD* } \\
Metabolic syndrome-related indicators & Medium-risk OR ${ }^{*}$ (95\% CI) & High-risk OR (95\% CI) \\
\hline WC $^{\mathrm{a}}$ & $1.54(.93-2.55)$ & $2.07(1.04-4.13)$ \\
$\mathrm{BP}^{\mathrm{b}}$ & $3.12(1.75-5.55)$ & $1.52(9.10-66.09)$ \\
$\mathrm{FPG}^{\mathrm{c}}$ & $1.34(.83-2.15)$ & $1.48(.74-2.35)$ \\
$\mathrm{TG}^{\mathrm{d}}$ & $1.86(1.11-3.13)$ & $4.03(2.03-7.98)$ \\
HDL-C & $1.36(.82-2.25)$ &
\end{tabular}

${ }^{\mathrm{a}} \mathrm{WC}$ : waist circumference.

${ }^{b} \mathrm{BP}$ : blood pressure.

${ }^{\mathrm{c}} \mathrm{FPG}$ : fasting plasma glucose.

${ }^{\mathrm{d}} \mathrm{TG}$ : triglyceride.

${ }^{\mathrm{e}} \mathrm{HDL}-\mathrm{C}$ : high-density lipoprotein cholesterol. * Using the low-risk $\leqq 10 \%$ group as the reference group. ${ }^{*}$ Odds ratios were adjusted for sex, age, smoking, alcohol consumption, and betel-nut chewing.

consumption, and betel-nut chewing, we found that subjects with abdominal obesity, elevated blood pressure, raised TG, and reduced HDL-C were nonetheless more likely to develop CVD within 10 years, compared to subjects without these disorders. The odds ratios for subjects with these disorders ranged from 1.86 to 24.52 times more likely to develop CVD.

One previous study demonstrated a relationship between blood pressure and mortality among men in different parts of the world. It revealed that the overall unadjusted relative risk of death because of coronary heart disease was 1.17 times (95\% CI, 1.14 to 1.20 ). When systolic blood pressure increased by $10 \mathrm{mmHg}$, it was 1.13 times (95\% CI, 1.10 to 1.15), and when diastolic blood pressure increased by $5 \mathrm{mmHg}$, it was 1.28 times for an increase in risk after adjustment for variability in blood pressure [15]. The INTERHEART study found that the ORs of myocardial infarction associated with abdominal obesity were 1.12 for top versus lowest tertile of waist circumference and 1.62 for middle versus lowest tertile of waist circumference [16]. Another study with Chinese adults indicated that an increase in waist circumference raised the risk of developing CVDs [17]. The Framingham Heart Study results showed that level of HDL-C was significant for determining CVD risk [18]. Furthermore, the Second National Health and Nutrition Examination Survey in the United States found that subjects who had even one or two metabolic syndrome components were at increased risk for mortality from CHD and CVD. The same study found that patients with metabolic syndrome combined with diabetes were at the highest risk for CVD (RR, 6.45; 95\% CI, 4.24-9.79) [19]. Similarly, a six-year followup study indicated that after adjusting for potential confounding factors, participants with metabolic syndrome were at a significantly higher risk for CVD and myocardial infarctions [20].

According to the results from these studies, we realized that metabolic syndrome-related indicators are associated with higher risk of CVDs. Certain studies have also shown that smoking and alcohol consumption increase the risk of developing a heart disease [21-23]. Our study found that betel-nut chewing, a special health risk factor in Taiwan, may also be a factor in 10-year risk of developing CVD. Similar results were found in a previous study [24-26].
Several reports mentioned that areca nut chewing may contribute to cardiovascular disease (mainly cerebrovascular) because certain chemicals from alkaloids (mainly arecoline and arecaidine) may trigger increased appetite and glucose intolerance $[27,28]$. Consequently, these factors may lead to obesity, diabetes, and subsequent death from cardiovascular conditions. In addition, the nut-chewing population may be at increased risk for CVD because of an increased oxidative stress $[29,30]$ and reduced $\gamma$-aminobutyric acid receptor activity. Specific arecal alkaloids, such as arecoline, are $\gamma$ aminobutyric acid receptor antagonists [31]. Other less wellcharacterized metabolic effects may also play a role.

Our study selected metabolic syndrome-related indicators and used multiple logistic regression to assess the 10year risk for CVD. Certain prospective epidemiologic studies have shown that the incidence of coronary events is reduced by raising HDL-C, which was an independent predictor of CVD [32, 33].

This study has limitations. First, it was designed as a cross-sectional study; thus, only the "snapshot" of association between metabolic syndrome-related indicators and 10year risk for CVD could be evaluated. Data took the form of prevalence rates only. The analysis was purely correlational, and, therefore, no causal inferences can be made. Second, our study did not collect sufficient information on medication use and dietary habits from the participants. It is possible that residual confounding by these factors may also affect the metabolic syndrome-CVD link.

In conclusion, the present study demonstrated that abdominal obesity, hypertension, and dyslipidemic components of metabolic syndrome correlated strongly with 10year risk for developing CVD. Therefore, it is mandatory for medical personnel to screen middle-aged and elderly population with metabolic syndrome for WC, hypertension, hypertriglyceridemia, and reduced HDL-C. Appropriate management of these problems can reduce the risk of CVD.

\section{Acknowledgments}

The authors are thankful to the physical examination assistance from Dr. Liang Tzu-An and Liao Yu-Kuei of Pingtung Christian Hospital and the other investigators, the staff, and 
the participants of the health screening program for their valuable contributions.

\section{References}

[1] R. H. Eckel, K. G. M. M. Alberti, S. M. Grundy, and P. Z. Zimmet, "The metabolic syndrome," The Lancet, vol. 375, no. 9710, pp. 181-183, 2010.

[2] M. A. Cornier, D. Dabelea, T. L. Hernandez et al., "The metabolic syndrome," Endocrine Reviews, vol. 29, no. 7, pp. 777$822,2008$.

[3] S. M. Grundy, "Metabolic syndrome: connecting and reconciling cardiovascular and diabetes worlds," Journal of the American College of Cardiology, vol. 47, no. 6, pp. 1093-1100, 2006.

[4] B. Isomaa, P. Almgren, T. Tuomi et al., "Cardiovascular morbidity and mortality associated with the metabolic syndrome," Diabetes Care, vol. 24, no. 4, pp. 683-689, 2001.

[5] J. M. Dekker, C. Girman, T. Rhodes et al., "Metabolic syndrome and 10-year cardiovascular disease risk in the Hoorn Study," Circulation, vol. 112, no. 5, pp. 666-673, 2005.

[6] S. Malik, N. D. Wong, S. S. Franklin et al., "Impact of the metabolic syndrome on mortality from coronary heart disease, cardiovascular disease, and all causes in United States adults," Circulation, vol. 110, no. 10, pp. 1245-1250, 2004.

[7] B. E. K. Klein, R. Klein, and K. E. Lee, "Components of the metabolic syndrome and risk of cardiovascular disease and diabetes in Beaver Dam," Diabetes Care, vol. 25, no. 10, pp. 1790-1794, 2002.

[8] W. H. Pan, W. T. Yeh, and L. C. Weng, "Epidemiology of metabolic syndrome in Asia," Asia Pacific Journal of Clinical Nutrition, vol. 17, no. 1, pp. 37-42, 2008.

[9] J. I. Cleeman, "Executive summary of the third report of the National Cholesterol Education Program (NCEP) expert panel on detection, evaluation, and treatment of high blood cholesterol in adults (adult treatment panel III)," Journal of the American Medical Association, vol. 285, no. 19, pp. 2486-2497, 2001.

[10] C. E. Tan, S. Ma, D. Wai, S. K. Chew, and E. S. Tai, "Can we apply the National Cholesterol Education Program adult treatment panel definition of the metabolic syndrome to Asians?" Diabetes Care, vol. 27, no. 5, pp. 1182-1186, 2004.

[11] L. C. Hwang, C. H. Bai, C. J. Chen, and K. L. Chien, "Gender difference on the development of metabolic syndrome: a population-based study in Taiwan," European Journal of Epidemiology, vol. 22, no. 12, pp. 899-906, 2007.

[12] A. M. J. Wassink, Y. van der Graaf, J. K. Olijhoek, and F. L. J. Visseren, "Metabolic syndrome and the risk of new vascular events and all-cause mortality in patients with coronary artery disease, cerebrovascular disease, peripheral arterial disease or abdominal aortic aneurysm," European Heart Journal, vol. 29, no. 2, pp. 213-223, 2008.

[13] W. H. H. Sheu, S. Y. Chuang, W. J. Lee, S. T. Tsai, P. Chou, and C. H. Chen, "Predictors of incident diabetes, metabolic syndrome in middle-aged adults: a 10-year follow-up study from Kinmen, Taiwan," Diabetes Research and Clinical Practice, vol. 74, no. 2, pp. 162-168, 2006.

[14] S. W. Lai, K. F. Liao, T. C. Li, H. F. Lin, W. Y. Lin, and C. H. Lin, "Metabolic syndrome in older people in Taiwan: a hospitalbased study," Internal Medicine Journal, vol. 36, no. 10, pp. 648-651, 2006.

[15] P. C.W. Van Den Hoogen, E. J.M. Feskens, N. J.D. Nagelkerke, A. Menotti, A. Nissinen, and D. Kromhout, "The relation between blood pressure and mortality due to coronary heart disease among men in different parts of the world," New England Journal of Medicine, vol. 342, no. 1, pp. 1-8, 2000.

[16] P. S. Yusuf, S. Hawken, S. Ounpuu et al., "Effect of potentially modifiable risk factors associated with myocardial infarction in 52 countries (the INTERHEART study): case-control study," Lancet, vol. 364, no. 9438, pp. 937-952, 2004.

[17] R. P. Wildman, D. Gu, K. Reynolds, X. Duan, X. Wu, and J. He, "Are waist circumference and body mass index independently associated with cardiovascular disease risk in Chinese adults?" American Journal of Clinical Nutrition, vol. 82, no. 6, pp. 11951202, 2005.

[18] P. W. F. Wilson, "High-density lipoprotein, low-density lipoprotein and coronary artery disease," American Journal of Cardiology, vol. 66, no. 6, pp. 7A-10A, 1990.

[19] S. Malik, N. D. Wong, S. S. Franklin et al., "Impact of the metabolic syndrome on mortality from coronary heart disease, cardiovascular disease, and all causes in United States adults," Circulation, vol. 110, no. 10, pp. 1245-1250, 2004.

[20] J. Butler, N. Rodondi, Y. Zhu et al., "Metabolic syndrome and the risk of cardiovascular disease in older adults," Journal of the American College of Cardiology, vol. 47, no. 8, pp. 1595-1602, 2006.

[21] J. A. Ambrose and R. S. Barua, "The pathophysiology of cigarette smoking and cardiovascular disease: an update," Journal of the American College of Cardiology, vol. 43, no. 10, pp. 17311737, 2004.

[22] O. Nilssen, M. Averina, T. Brenn, J. Brox, A. Kalinin, and V. Archipovski, "Alcohol consumption and its relation to risk factors for cardiovascular disease in the north-west of Russia: the Arkhangelsk study," International Journal of Epidemiology, vol. 34, no. 4, pp. 781-788, 2005.

[23] P. H. Whincup, J. A. Gilg, J. R. Emberson et al., "Passive smoking and risk of coronary heart disease and stroke: prospective study with cotinine measurement," British Medical Journal, vol. 329, no. 7459, pp. 200-204, 2004.

[24] T. Y. Lan, W. C. Chang, Y. J. Tsai, Y. L. Chuang, H. S. Lin, and T. Y. Tai, "Areca nut chewing and mortality in an elderly cohort study," American Journal of Epidemiology, vol. 165, no. 6, pp. 677-683, 2007.

[25] A. M. F. Yen, L. S. Chen, Y. H. Chiu, B. J. Boucher, and T. H. H. Chen, "A prospective community-population-registry-based cohort study of the association between betel-quid chewing and cardiovascular disease in men in Taiwan (KCIS no. 19)," American Journal of Clinical Nutrition, vol. 87, no. 1, pp. 7078, 2008.

[26] W. Y. Lin, T. Y. Chiu, L. T. Lee, C. C. Lin, C. Y. Huang, and K. C. Huang, "Betel nut chewing is associated with increased risk of cardiovascular disease and all-cause mortality in Taiwanese men," American Journal of Clinical Nutrition, vol. 87, no. 5, pp. 1204-1211, 2008.

[27] T. H. Tung, Y. H. Chiu, L. S. Chen, H. M. Wu, B. J. Boucher, and T. H. H. Chen, "A population-based study of the association between areca nut chewing and Type 2 diabetes mellitus in men (Keelung Community-based Integrated Screening programme No. 2)," Diabetologia, vol. 47, no. 10, pp. 17761781, 2004.

[28] W. C. Chang, C. F. Hsiao, H. Y. Chang et al., "Betel nut chewing and other risk factors associated with obesity among Taiwanese male adults," International Journal of Obesity, vol. 30, no. 2, pp. 359-363, 2006.

[29] K. C. Lai and T. C. Lee, "Genetic damage in cultured human keratinocytes stressed by long-term exposure to areca nut extracts," Mutation Research, vol. 599, no. 1-2, pp. 66-75, 2006. 
[30] B. Molavi and J. L. Mehta, "Oxidative stress in cardiovascular disease: molecular basis of its deleterious effects, its detection, and therapeutic considerations," Current Opinion in Cardiology, vol. 19, no. 5, pp. 488-493, 2004.

[31] D. Z. Hung and J. F. Deng, "Acute myocardial infarction temporally related to betel nut chewing," Veterinary and Human Toxicology, vol. 40, no. 1, pp. 25-28, 1998.

[32] J. P. Després, I. Lemieux, G. R. Dagenais, B. Cantin, and B. Lamarche, "HDL-cholesterol as a marker of coronary heart disease risk: the Québec cardiovascular study," Atherosclerosis, vol. 153, no. 2, pp. 263-272, 2000.

[33] H. B. Rubins, S. J. Robins, D. Collins et al., "Gemfibrozil for the secondary prevention of coronary heart disease in men with low levels of high-density lipoprotein cholesterol," $\mathrm{New}$ England Journal of Medicine, vol. 341, no. 6, pp. 410-418, 1999. 


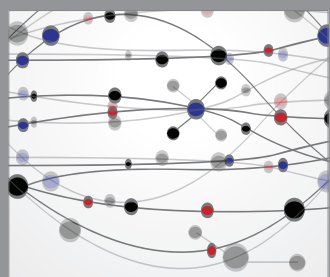

The Scientific World Journal
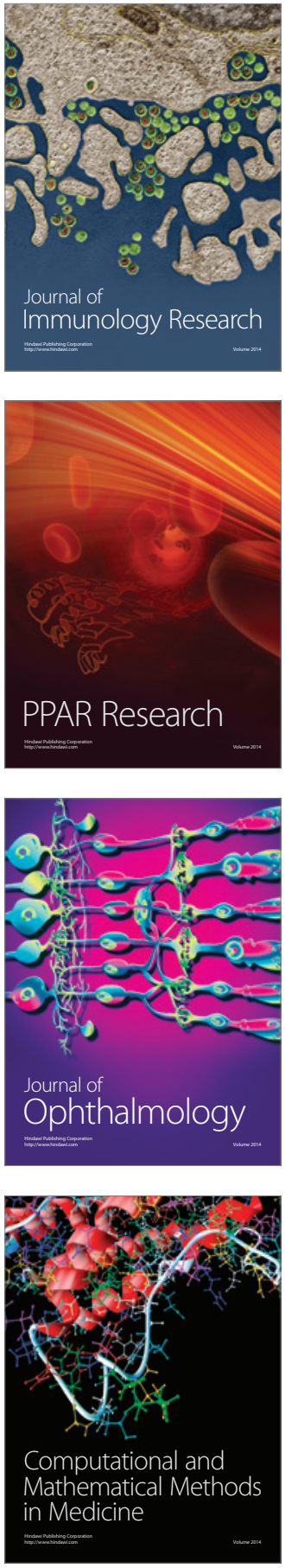

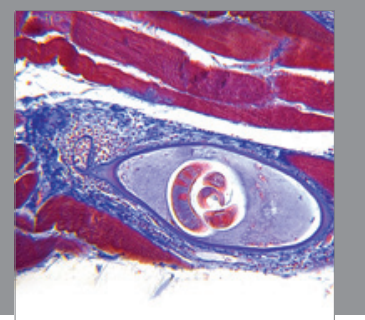

Gastroenterology

Research and Practice
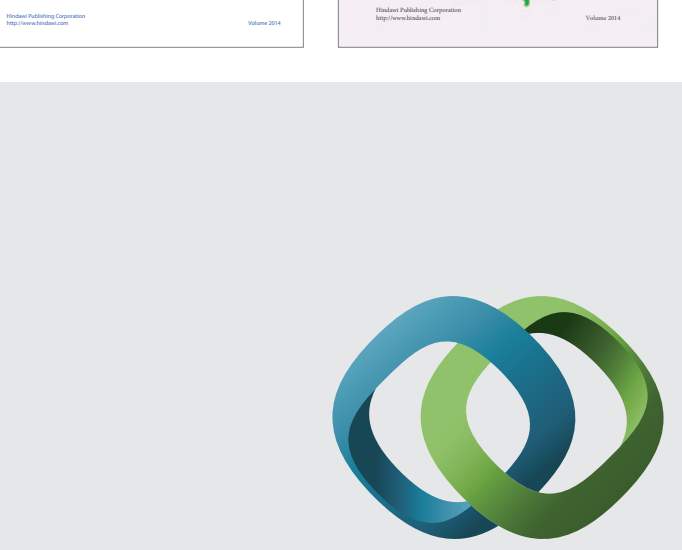

\section{Hindawi}

Submit your manuscripts at

http://www.hindawi.com
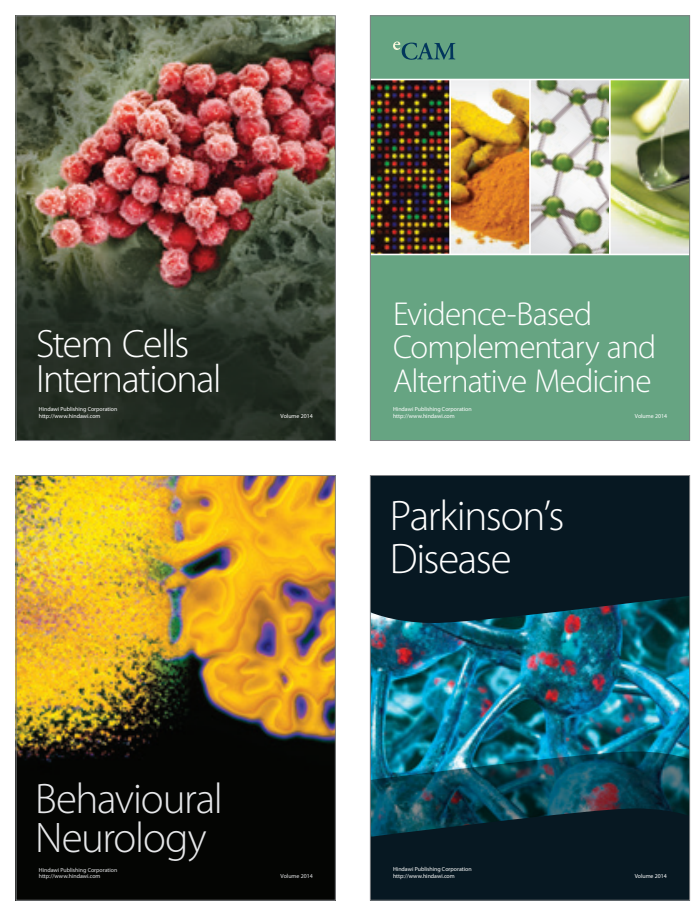

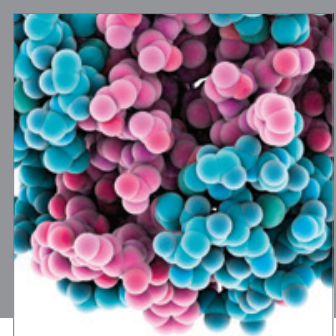

Journal of
Diabetes Research

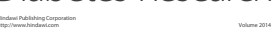

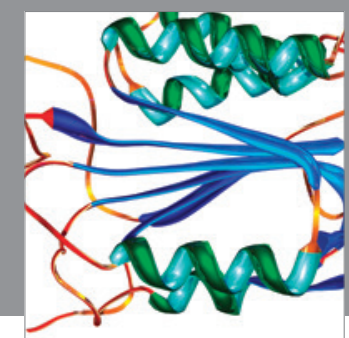

Disease Markers
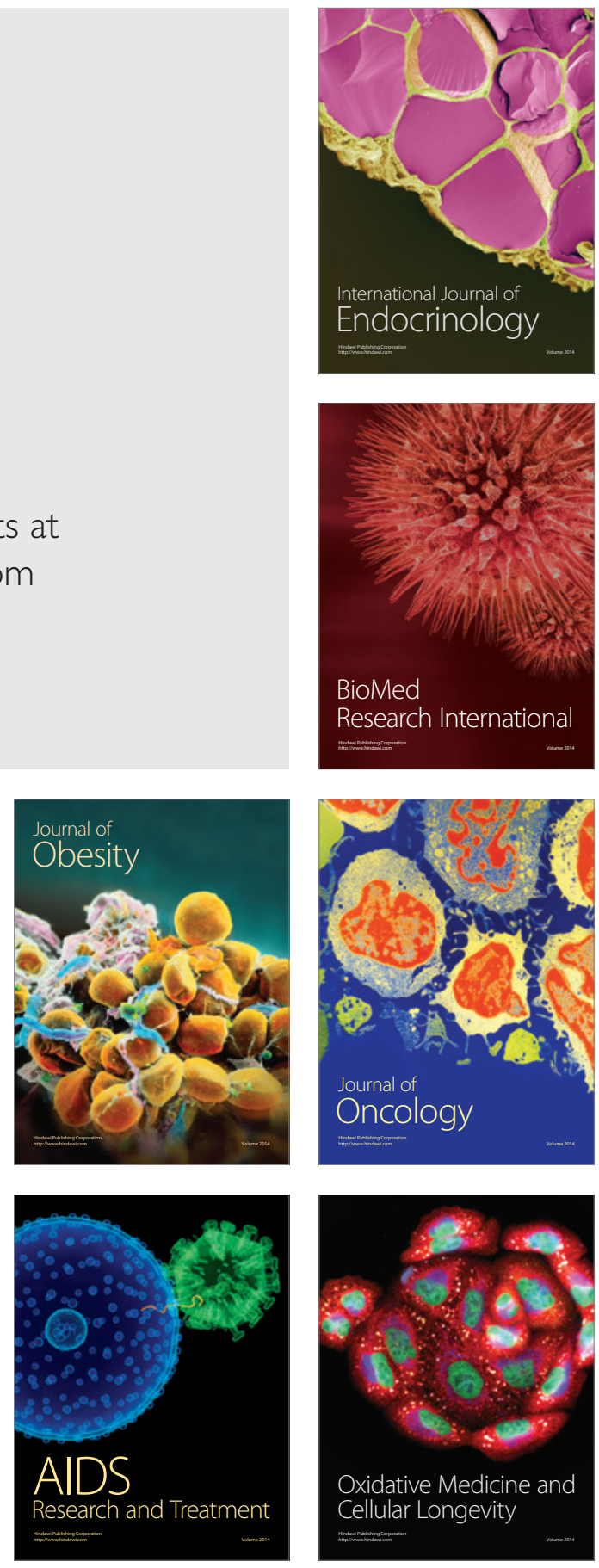